\title{
LA INCORPORACIÓN DE LOS DERECHOS HUMANOS A LAS METODOLOGÍAS DE COOPERACIÓN AL DESARROLLO A TRAVÉS DEL ENFOQUE DE DERECHOS Y LAACCIÓN SIN DAÑO
}

\section{THE INTEGRATION OF HUMAN RIGHTS INTO THE METHODOLOGIES OF DEVELOPMENT COOPERATION THROUGH THE HUMAN-RIGHTS-BASED APPROACH (HRBA) AND THE DO NO HARM APPROACH,}

\author{
Alfredo Langa Herrero \\ Alice Salomon University, Berlin, Alemania \\ alfredo.langa@iecah.org
}

Recibido: abril de 2019

Aceptado: diciembre de 2019

Palabras clave: Cooperación internacional, Desarrollo, Enfoque de Marco Lógico, Acción sin Daño, Enfoque de Derechos Humanos.

Keywords: International cooperation, Development, Logical Framework Approach, Do no Harm, HumanRights-Based Approach.

Resumen: En este artículo se presentan herramientas concretas para incorporar el Enfoque Basado en los Derechos Humanos (EBDH) en los diferentes niveles de planificación de las iniciativas de desarrollo. Éstas necesitan de unidades de planificación cercanas a los ciudadanos, como son los proyectos, por lo que es fundamental que el EBDH sea tenido en cuenta en la toma de decisiones, de arriba hacia abajo y de abajo hacia arriba. Para ello, se propone la incorporación de la metodología de la Acción sin Daño, desarrollada por Mary B. Anderson, al ciclo de proyecto de desarrollo y al enfoque de marco lógico.

Abstract: This article introduces specific tools in order to include the HumanRights-Based Approach (HRBA) in the different levels of development planning. The planning units closer to the citizens - the projects - need the HRBA to be taken into consideration for the decision making process, bottom up as well as from the top to bottom. In doing so, the Do no Harm approach, developed by Mary B. Anderson, is proposed to be incorporated in the project cycle managed by the Logical Framework Approach. 


\section{l. Introducción}

Este artículo no tiene por objetivo analizar el concepto de desarrollo o sus diversas teorías y debates desde un punto de vista teórico y "macro", sino que presenta una metodología de gestión del ciclo del proyecto fruto de la combinación de métodos existentes. En concreto se combina la metodología del Enfoque de Marco Lógico y la Acción sin Daño y se esboza cómo podrían trabajar en conjunto para el caso de proyectos de desarrollo, teniendo en cuenta el denominado Enfoque Basado en los Derechos Humanos (EBDH).

Este texto no pretende explicar detalladamente ambas metodologías y dicho enfoque, sino presentar una manera de combinarlas de manera práctica. Por ello, la primera parte introduce al lector en el $\mathrm{EBDH}$, para mostrar, posteriormente, una aproximación al ciclo del proyecto e introducir la metodología del marco lógico de manera breve, y respecto a las fases de identificación y diseño del proyecto. Posteriormente, se entrará a delinear la Acción sin Daño para presentar una combinación de ambas focalizada en las fases de identificación y diseño del proyecto, que permita contener el EBDH de manera práctica.

\section{El Enfoque Basado en los Derechos Humanos (EBDH)}

Según la Oficina del Alto Comisionado de las Naciones Unidas para los Derechos Humanos (OHCHR, 2006: 15): "El enfoque basado en los derechos humanos es un marco conceptual para el proceso de desarrollo humano que desde el punto de vista normativo está basado en las normas internacionales de derechos humanos, y desde el punto de vista operacional está orientado a la promoción y la protección de los derechos humanos. Su propósito es analizar las desigualdades que se encuentran en el centro de los problemas de desarrollo y corregir las prácticas discriminatorias y el injusto reparto del poder que obstaculizan el progreso en materia de desarrollo". Por ello, desde el enfoque y la perspectiva de los derechos humanos, la planificación del desarrollo económico y social no es producto de un sentimiento caritativo, sino el resultado de un sistema de derechos y de los consecuentes deberes que dichos derechos establecen. De esta manera, si hablamos de enfoque de derechos en la cooperación internacional al desarrollo, la solidaridad internacional no es la base de las políticas, planes, programas y proyectos de desarrollo, sino la realización de los derechos humanos de aquellos a los que se dirigen dichas políticas, planes, programas y proyectos (UNFPA, 2010; OHCHR, 2006).

Esto implica la identificación de los sujetos de los derechos humanos, así como a aquellas instituciones que tienen la obligación de hacer que los derechos humanos se cumplan. De esta manera: "Un enfoque basado en los derechos humanos identifica a los titulares de derechos y aquello a lo que tienen derecho, y a los correspondientes titulares de deberes y las obligaciones que les incumben, y procura fortalecer la capacidad de los titulares de derechos para reivindicar éstos y de los titulares de deberes para cumplir sus obligaciones" (OHCHR, 2006: 15). Por lo tanto, la cooperación internacional al desarrollo se basa, no sólo en las políticas y los planes de desarrollo de los organismos ejecutores y financiadores, sino en los principios y las normas de la legislación internacional en materia de 


\section{Tabla l. Los enfoques de cooperación internacional}

\begin{tabular}{|ll|}
\hline Enfoque de necesidades & EBDH \\
\hline Se centra en el resultado & Se centra en el proceso. \\
\hline $\begin{array}{l}\text { Pone énfasis en la satisfacción de las } \\
\text { necesidades }\end{array}$ & $\begin{array}{l}\text { Pone el énfasis en aumentar realización } \\
\text { de derechos }\end{array}$ \\
\hline $\begin{array}{l}\text { Reconoce los derechos como reclamos } \\
\text { válidos }\end{array}$ & $\begin{array}{l}\text { Reconoce los derechos humanos } \\
\text { individuales y colectivos como reclamos } \\
\text { ante los titulares de obligaciones }\end{array}$ \\
\hline $\begin{array}{l}\text { Los individuos son objeto de las } \\
\text { intervenciones de desarrollo }\end{array}$ & $\begin{array}{l}\text { Los individuos y grupos son empoderados } \\
\text { para reclamar sus derechos }\end{array}$ \\
\hline Los individuos merecen asistencia & $\begin{array}{l}\text { Los individuos tienen derecho a la } \\
\text { asistencia }\end{array}$ \\
\hline $\begin{array}{l}\text { Se centra en las causas inmediatas de los } \\
\text { problemas }\end{array}$ & $\begin{array}{l}\text { Se centra en las causas estructurales de } \\
\text { los problemas y sus manifestaciones }\end{array}$ \\
\hline
\end{tabular}

Fuente: Fernández Aller, Celia et alia (2009:59).

derechos humanos (Gómez Galán et alii, 2013; OHCHR, 2006).

Por todo ello, se identifican, al menos dos enfoques a la hora de gestionar el ciclo de los programas y proyectos de desarrollo. El EBDH, que se ha esbozado, y un enfoque basado en las necesidades, que es el que generalmente se ha aplicado en las últimas décadas. Estos dos enfoques se resumen en el cuadro anterior.

La necesidad de incorporar este enfoque a la cooperación internacional suscitó la celebración en 2003 del segundo seminario interinstitucional relativo al EBDH, en la ciudad norteamericana de Stamford, con el objetivo de alcanzar "un entendimiento común entre los organismos de las Naciones Unidas" (OHCHR, 2006: 35). En el seminario se identificaron tres elementos fundamentales a tener en cuenta en los objetivos de los programas y proyectos de cooperación al desarrollo:

- que éstos se dirijan a la realización de los derechos;

- que sean regidos por los estándares y principios de derechos humanos en todos los sectores y áreas de trabajo y en todas las fases del ciclo de planificación;
- y que dichos objetivos hagan posible y refuercen el impulso de la capacidad de los titulares de derechos de reclamar sus derechos, y de los titulares de deberes de hacer frente a sus obligaciones.

En este sentido, los titulares de derechos son aquellas personas o grupos de personas cuyos derechos han de ser protegidos por las leyes nacionales e internacionales de derechos humanos. Por ello, los programas y proyectos de cooperación han de velar porque los derechos humanos de los beneficiarios de las iniciativas sean garantizados sin distinción alguna, especialmente de aquellos grupos en condiciones de pobreza, vulnerabilidad o exclusión (Fernández Aller, Celia et alia, 2009).

Por otro lado, los titulares de deberes u obligaciones son aquellos actores que tienen la obligación de respetar, proteger y realizar los derechos de los titulares de derechos, ya que dichos derechos implican un deber para los portadores de deberes, valga la redundancia. Independientemente del tipo de programa o proyecto de cooperación internacional, el portador de deberes puede ser tanto un Estado, una organización internacional o un actor no estatal, como una 
ONG o incluso un individuo. No obstante, el Estado supone el principal portador de deberes y la legislación nacional debe definir la manera en que sus obligaciones, respecto a los derechos humanos, se consumarán a nivel nacional, regional y local, así como la manera en que los individuos, las empresas, los Gobiernos regionales y locales, las ONG y otros actores de la sociedad asumen la responsabilidad de la ejecución (UNFPA, 2010; OHCHR, 2006). Por ello, a estos últimos agentes se les denomina titulares de responsabilidades desde el $\mathrm{EBDH}$, ya que las obligaciones pertenecen normalmente al Estado y las responsabilidades contienen un aspecto moral y ético. Los actores titulares de responsabilidades son responsables de la realización, promoción y protección de los derechos humanos, ya que sus actividades por acción u omisión tienen un impacto sobre el ejercicio de los derechos humanos, sobre todo de la población más vulnerable o excluida socialmente (Fernández Aller, Celia et alii, 2009). De esta manera, es el titular de responsabilidades, sea éste una organización internacional o local el que "carga" con la responsabilidad moral y ética de la realización de los derechos humanos, por lo que la metodología que utilice para gestionar el ciclo del programa o proyecto influirá en el ejercicio de dicha responsabilidad. En este texto será el proyecto el protagonista del proceso de planificación y será su ciclo el que se bosqueja seguidamente.

\section{La gestión del ciclo del proyecto}

El proyecto se considera, en el mundo de la planificación, como la acción planificada más cercana a la realidad y a los actores que intervienen en la misma. El proyecto se definiría como un conjunto de actividades o tareas que son llevadas a cabo ordenadamente en un periodo de tiempo determinado y que tienen objetivos comunes. Representa, por tanto, una intervención que está limitada en el tiempo y también en el espacio. Si nos referimos, además, al proyecto de desarrollo, el objetivo del conjunto de actividades tiene que lograr dicho desarrollo, por lo que los gestores del proyecto han de tener claro desde un principio el enfoque de desarrollo que desean trasmitir. Concretamente el proyecto ha sido definido por la Comisión Europea como: "una serie de actividades dirigidas hacia el cumplimiento de objetivos claramente especificados, en un periodo de tiempo determinado y con un presupuesto definido" (Europe Aid Cooperation Office, 2004: 8).

De esta manera, habría que acudir a algunas de las perspectivas de desarrollo que comentan Arturo Escobar (2014) o Aram Ziai (2016) respecto a las tesis liberales, marxistas o postestructuralistas, para conocer el destino del proyecto, respecto al desarrollo que pretende alcanzar. No obstante, independientemente del enfoque de desarrollo, el proyecto tiene un ciclo de vida que implica una serie de fases que han de ser gestionadas en función de ciertas metodologías. Tomando las aproximaciones de la Agencia Noruega de Cooperación Internacional (NORAD, 1999) o de la Unión Europea (EuropeAid Cooperation Office, 2004), el ciclo de vida del proyecto supone la consecución de una fase de identificación, otra de diseño o formulación, una fase de ejecución y seguimiento, así como una última fase de evaluación del proyecto, tal y como ilustra la siguiente figura. En este sentido, la definición de proyecto de la Comisión Europea establece que: "un proyecto ha de tener en cuenta los siguientes elemen- 


\section{Figura l. Ciclo de vida del proyecto.}
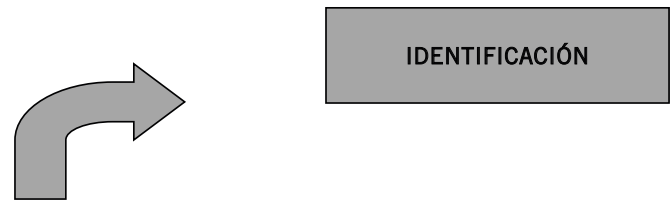

EVALUACIÓN


EJECUCIÓN/

MONITOREO

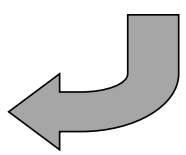

Fuente: Europe Aid Cooperation Office (2004) y elaboración propia.

tos: una clara identificación de actores, incluyendo los principales grupos objetivo y los beneficiarios finales; claros acuerdos de coordinación, gestión y financiación; un sistema de seguimiento y evaluación; así como, un nivel apropiado de análisis financiero y económico de manera que esté claramente indicado que los beneficios del proyecto excederán a sus costes" (Europe Aid Cooperation Office, 2004: 8).

La fase de identificación se centra en la descripción, caracterización y análisis del contexto donde se llevará a cabo el proyecto, haciendo hincapié en los diferentes actores y las problemáticas sobre las que se pretende actuar, proponiendo soluciones y esbozando los principales elementos del proyecto. De manera paralela, la fase de diseño o formulación toma los elementos de la fase de identificación y los sistematiza, para de esta manera, generar un cuerpo ordenado centrado en unos objetivos, con el que pasar a la ejecución del proyecto donde las actividades se im- plementan y monitorean. Finalmente, la fase de evaluación corresponde a una valoración respecto al logro de los objetivos del proyecto, entre otros elementos.

La gestión de cada fase del ciclo de vida del proyecto se lleva a cabo mediante diferentes metodologías, de las cuales, una de las más ampliamente aplicadas es la metodología denominada Enfoque de Marco Lógico (EML), nacida en el seno de la Agencia de Cooperación de EEUU (USAID) y desarrollada por la Agencia de Cooperación Técnica Alemana (GTZ) y la Agencia Noruega de Cooperación (NORAD). Esta metodología es usada, igualmente, por la Comisión Europea y los Estados miembros de la UE, y está incluida en sus principales manuales de gestión de proyectos (Ortegón, Edgar et alia, 2005).

Este texto se enfoca en las fases de identificación y diseño, en las que la metodología del EML sigue los pasos que muestra la figura 2, para en apartados posteriores com- 
Figura 2. Ciclo de vida del proyecto y Enfoque de Marco Lógico

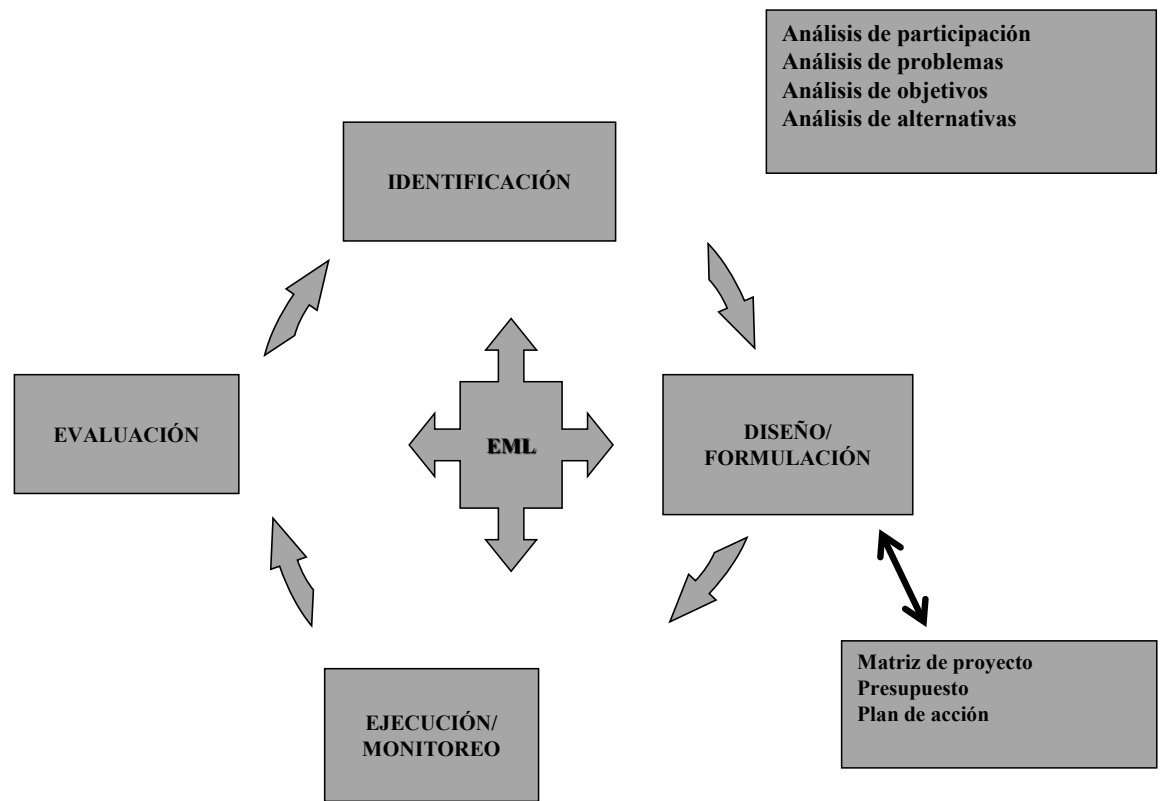

Fuente: Europe Aid Cooperation Office (2004) y elaboración propia.

binar sus etapas con las de la metodología de la Acción sin Daño. En cualquier caso, el actor 0 actores encargado de gestionar el ciclo de proyecto mediante el EML se convierte en titular de responsabilidades según el EBDH, por lo que la manera en que lleve a cabo el análisis de participación, el análisis de problemas, el análisis de objetivos o el de alternativas influirá en la realización de los derechos humanos de los beneficiarios de las iniciativas.

\section{El Enfoque de Marco Lógico (EML) y el ciclo del proyecto}

Como se ha visto, durante la fase de identificación del proyecto, el EML se articula en torno a cuatro pasos que suponen analizar a los actores, instituciones, organizaciones o individuos relevantes para el futuro proyecto; analizar los problemas de algunos de estos actores; analizar las soluciones a los problemas anteriores, siempre que fuera posible; así como seleccionar y analizar alternativas o estrategias de acción (NORAD, 1999; EuropeAid Cooperation Office, 2005; Ortegón, Edgar et alia, 2005). Por tanto, estos pasos implican los siguientes análisis:

- El análisis de los actores o análisis de participación.

- El análisis de los problemas.

- El análisis de los objetivos.

- El análisis de las alternativas o las estrategias.

Mediante el análisis de los actores o análisis de participación se identifican los principales actores del proyecto y se eligen aquellos que se beneficiarán de los resultados del mismo, así como a posibles socios. Tradicionalmente se diferencia entre beneficiarios directos e 
Figura 3. Ejemplo de árbol de problemas

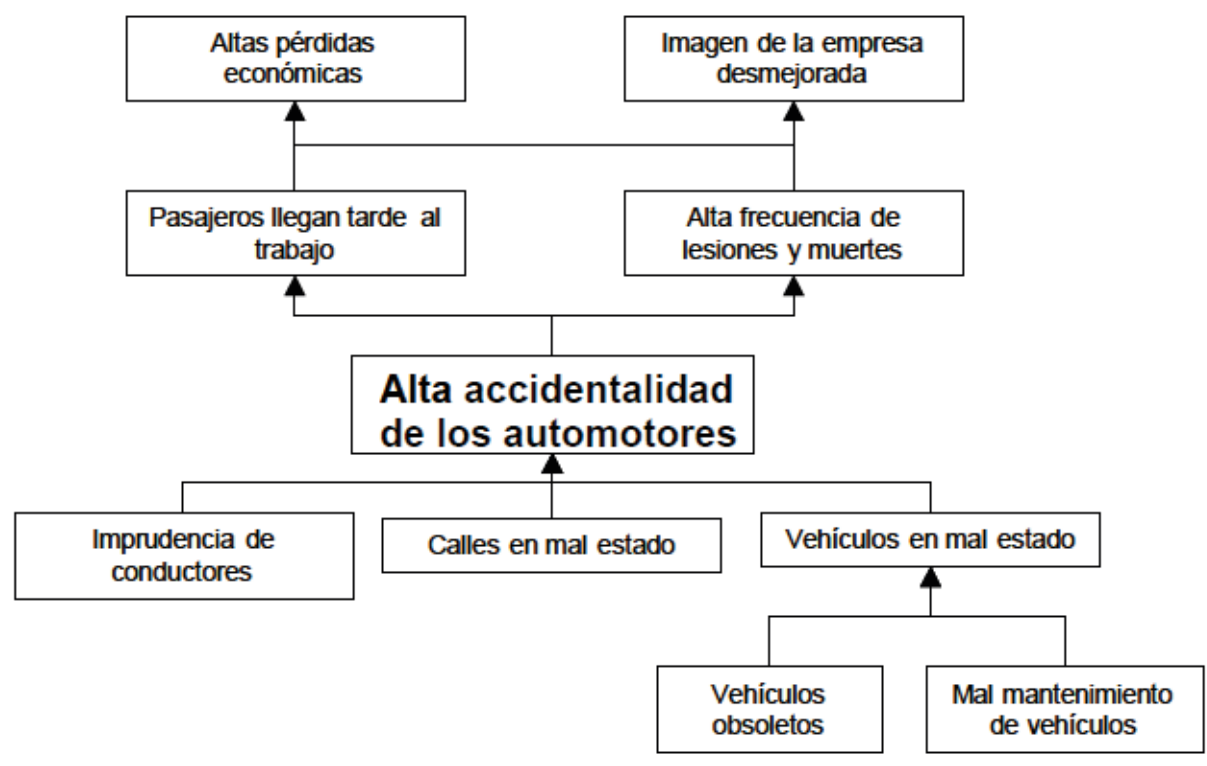

Fuente: Ortegón, Edgar et alia (2005: 95).

indirectos, actores neutrales y oponentes al proyecto, y se supone que el desarroIlo a alcanzar por dicho proyecto beneficiará a los intereses de los beneficiarios. De manera concreta, el análisis puede tener el formato de una matriz de actores donde se especifiquen sus potenciales, problemas y las relaciones con el resto de actores, o también puede ser presentado como un análisis de redes, a través de un mapa de actores o un diagrama de Venn (NORAD, 1999; EuropeAid Cooperation Office, 2005; Ortegón, Edgar et alia, 2005).

El análisis de los problemas, por su parte, se basa en la caracterización de las situaciones negativas que los beneficiarios identificados anteriormente afrontan. Este análisis se presenta en forma de árbol de problemas, donde la relación entre cada problema es de causa-efecto como muestra el ejemplo de la figura 3.
En el árbol anterior cada recuadro representa un problema, que es al mismo tiempo causa y efecto de otro, con una lógica desde abajo hacia arriba. De esta manera, el problema "Alta accidentalidad de los automotores" es la causa de los problemas "Pasajeros llegan tarde al trabajo" y "Alta frecuencia de lesiones y muertes", y a la vez es el efecto de los problemas "Imprudencia de Conductores", "Calles en mal estado" y "Vehículos en mal estado" (Ortegón, Edgar et alia, 2005). La aproximación del EML se antoja neutral con respecto a la realización de los derechos humanos, por cuanto a que los problemas son situaciones negativas o necesidades, pero los beneficiarios no son titulares de derechos, sino "titulares de necesidades", como se vio en la tabla 1.

El análisis de los objetivos, por el contrario, trata de convertir en soluciones los problemas anteriores, siempre que dicha 
Figura 4. Ejemplo de árbol de objetivos

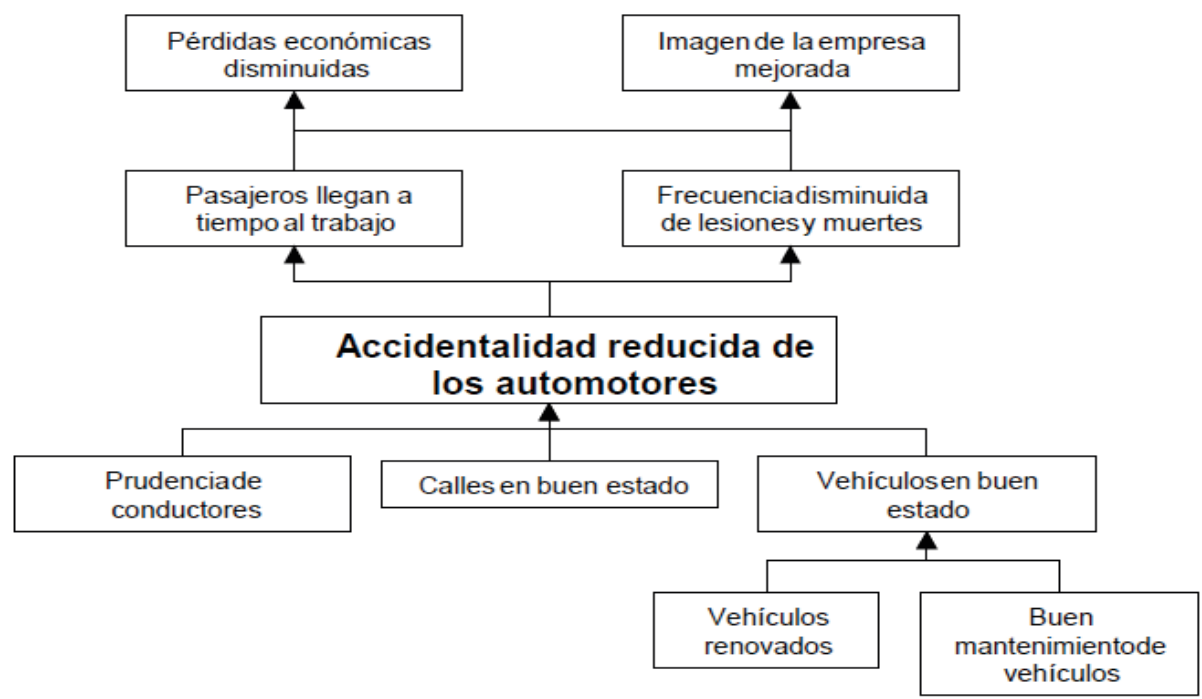

Fuente: Ortegón, Edgar et alii (2005: 96).

solución sea realista y posible, por lo que el árbol de problemas se transforma en un árbol de objetivos. La lógica de abajo hacia arriba se mantiene, pero cambia a una lógica medio-fin. Es decir, cada recuadro es al mismo tiempo medio y fin (Ortegón, Edgar et alia, 2005).

El problema "Alta frecuencia de lesiones y muertes" se transforma en la solución/ objetivo "Accidentalidad reducida de los automotores", que constituye el medio para alcanzar los fines "Pasajeros llegan a tiempo al trabajo" y "Frecuencia disminuida de lesiones y muertes". A su vez, los objetivos "Prudencia de conductores", "Vehículos en buen estado" y "Calles en buen estado" tienen como fin la "Accidentalidad reducida de los automotores" (Ortegón, Edgar et alii, 2005).

Respecto al análisis de las alternativas o estrategias, éste supone el cuarto y último paso de la fase de identificación del pro- yecto y consiste en la elección de alguno o algunos de los ramales del árbol de objetivos. En resumen, la estrategia dependerá de cuál de los medios se pueden ejecutar o lograr con los recursos disponibles (NORAD, 1999; EuropeAid Cooperation Office, 2005; Ortegón, Edgar et alia, 2005). En el ejemplo de las figuras 4 y 5 , cada ramal implica una estrategia enfocada en la prudencia de los conductores, en la mejora de las calles o en el estado de los vehículos. El proceso de elección de una de estas opciones o de varias de ellas constituye el eje del análisis de alternativas o estrategias.

Una vez que estos análisis se han llevado a cabo, los principales elementos necesarios para el diseño de un proyecto son identificados, la fase de identificación se solapa con la fase de diseño o formulación, la cual culmina con la elaboración de una matriz de planificación, como 
Figura 5. Ejemplo de alternativas y elementos para la matriz

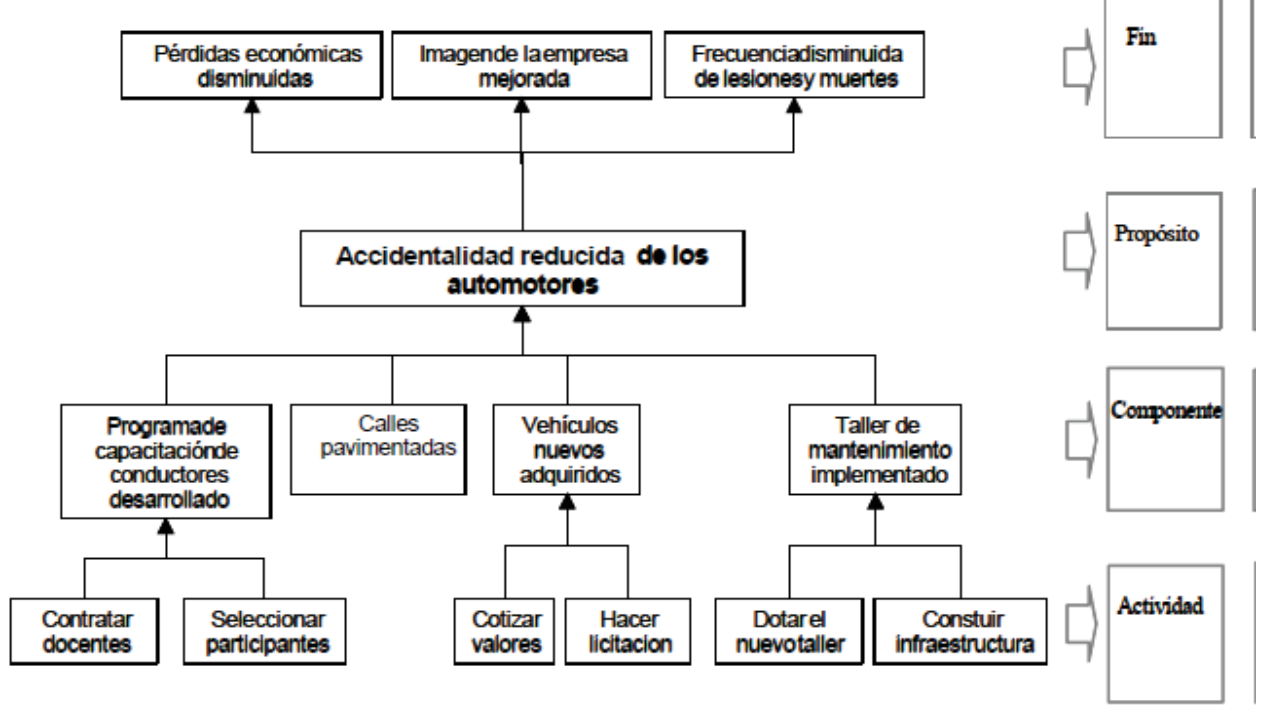

Fuente: Ortegón, Edgar et alia (2005: 96).

principal componente, aunque no el único, del diseño del proyecto. La matriz se fundamenta en los resultados del análisis de alternativas, que proporciona la información esencial y lógica necesaria para elaborar dicha matriz (NORAD, 1999; EuropeAid Cooperation Office, 2005; Ortegón, Edgar et alia, 2005).

Posteriormente, y ya entrados plenamente en la fase de diseño del proyecto, la información que suministra el análisis de alternativas es fundamental para identificar los elementos de la matriz de planificación. Como se ve en la figura 5 alguno de los objetivos del árbol pueden pasar a ser elementos de la matriz. Estos elementos definen el proyecto, así como el tipo de objetivo de desarrollo que se quiere lograr. Su primera columna (ver tabla 2) constituye el eje vertebrador del proyecto y debe definirse a partir de los análisis del marco lógico (NORAD, 1999; EuropeAid
Cooperation Office, 2005; Ortegón, Edgar et alia, 2005).

La siguiente tabla muestra un modelo simple de matriz de planificación con una primera columna que incluye actividades, resultados, objetivo específico y objetivo general. El desarrollo, por tanto, dependerá de que las actividades se realicen, los resultados se logren y el objetivo específico de alcance, contribuyendo, de este modo a la consecución de un objetivos general que necesita de más proyectos para su logro completo. Esta lógica de abajo hacia arriba constituye la denominada lógica de la intervención y debe ser examinada continuamente para comprobar su validez y factibilidad (NORAD, 1999; EuropeAid Cooperation Office, 2005; Ortegón, Edgar et alia, 2005).

Con la matriz se lograría elaborar un aspecto fundamental del diseño de un proyecto, como es la matriz de planificación. 
Tabla 2. Matriz de planificación de proyecto.

\begin{tabular}{|l|c|c|c|c|}
\hline & $\begin{array}{c}\text { Resumen } \\
\text { descriptivo }\end{array}$ & $\begin{array}{c}\text { Indicadores } \\
\text { objetivamente } \\
\text { verificables }\end{array}$ & $\begin{array}{c}\text { Fuentes de } \\
\text { verificación }\end{array}$ & $\begin{array}{c}\text { Factores de } \\
\text { Riesgo o } \\
\text { externos }\end{array}$ \\
\hline Objetivo general & & & & \\
\hline Objetivo específico & & & & \\
\hline Resultados & \multicolumn{2}{|c|}{ Costes } \\
\hline Actividades & \multicolumn{2}{|c|}{ Recursos } & \multicolumn{2}{c|}{} \\
\hline
\end{tabular}

Fuente: NORAD (1999) y elaboración propia.

A esta habría que unir, como aparece en la figura 2, un cronograma o plan de acción y un presupuesto económico, elementos fundamentales para comenzar a implementar un proyecto.

\section{Proyectos y acción sin daño.}

Que la matriz de planificación y, por tanto el proyecto, contenga un EBDH depende de los titulares de responsabilidades que gestionan y llevan a cabo las fases de identificación y diseño, por lo que las metodologías juegan un papel fundamental. La matriz anterior se ha elaborado mediante una metodología que no tiene en cuenta el contexto específico de violencia que puedan vivir los actores de un proyecto. Por ello, desde este texto se aboga por combinar la metodología de EML con la metodología de Acción sin Daño como estrategia para poner en práctica el EBDH, sobre todo porque ser "sensible" al conflicto y la violencia implica tener en cuenta las posible situaciones en que los derechos humanos podrían ser violentados.

La Acción sin Daño nació como una de las metodologías sensibles al conflicto, dentro del mundo de la acción humanitaria y no del desarrollo, sobre todo debido a la proliferación y el avance de las llamadas emergencias políticas complejas, en las que un conjunto de factores sociales, políticos, económicos, étnicos, religiosos y/o culturales, entre otros, han incidido y provocado situaciones de crisis humanitarias. Las emergencias políticas complejas implican crisis con múltiples causas, que hunden sus raíces en el propio sistema económico y en la organización política y social de los países que las sufren. Son crisis que tienen causas internas y de carácter internacional que complican la resolución del conflicto, en las cuales además, las partes beligerantes no están claramente definidas y que, en la mayor parte de las ocasiones, se benefician del escenario de conflicto (Wallace, 2016).

Las denominadas herramientas sensibles al conflicto han sido impulsadas a partir de los años noventa, con la desaparición de la política de bloques y el mayor peso conferido a las NNUU. En este contexto, las ONG y las agencias internacionales han aumentado su presencia y acción en escenarios de conflicto armado, pasando a ser un importante actor del propio conflicto, por lo que se ha presentado la necesidad de desarrollar enfoques que adecúen la ayuda prestada al contexto de violencia y, de esta manera, no convertir la ayuda en un elemento de conflicto, sino en un factor de paz (Wallace, 2016; Anderson, 1999). Por ello, desde el mundo de la acción humanitaria han surgido herramientas que tienen 
en cuenta las situaciones de violencia y, sobre todo, el escenario que presentan de las emergencias políticas complejas.

En esta línea, a principios de los años noventa Mary B. Anderson (1999 y 2009) planteó un interesante enfoque denominado Do no harm - no dañar o no hacer daño - basado en la práctica de varias ONG en diversos conflictos en todo el mundo, el cual supone una de las herramientas sensibles al conflicto más reconocidas. Estas herramientas de acción humanitaria sensibles al conflicto, hacen referencia a aquellos instrumentos o enfoques que tienen en cuenta la situación de conflicto armado y violencia en la que se encuentra la población destinataria de la ayuda, de cara a que dicha ayuda no provoque la prolongación o la intensificación del conflicto y la violencia.

El enfoque de la Acción sin Daño se lanzó en 1994 como iniciativa de la ONG norteamericana Collaborative for Development Action (CDA) y fue propuesto para analizar las interacciones entre el contexto del conflicto y las intervenciones y proyectos de acción humanitaria. Pretende neutralizar o disminuir los factores que agudizan un conflicto y fortalecer los factores que promuevan una solución no violenta del mismo, por lo que no trata sólo de evitar causar daño durante la ejecución de proyectos de ayuda sino, en la medida de lo posible, contribuir directa o indirectamente, además, a la construcción de la paz (Wallace, 2016).

Las Acción sin daño hace hincapié en los efectos positivos y negativos del comportamiento propio en situaciones de conflicto, aprovechando las conclusiones sacadas de las experiencias negativas de anteriores intervenciones humanitarias. Se emplea, sobre todo, en aquellos proyectos que no tratan directamente el conflicto, sino que quieren reaccionar de manera sensible al mismo, es decir, que quieren actuar en un contexto de conflicto teniendo en cuenta las circunstancias de éste (Wallace, 2016).

La Acción sin Daño constituye una herramienta importante para mejorar la planificación y la ejecución de intervenciones sensible ante conflictos y es parte de los enfoques para la prevención de las crisis. Se basa en el análisis, en cada caso, de lo que Anderson (2009 y 2009) llama "capacidades locales para la paz" y en tratar de centrar los proyectos de cooperación y acción humanitaria en torno a estas capacidades y en torno a lo que pueden ser mecanismos de comunicación y conexión entre comunidades en conflicto, evitando aquellos otros factores susceptibles de agravarlo (Wallace, 2016).

La Acción Sin Daño como metodología o herramienta sigue una serie de etapas que definen su marco analítico o de trabajo y que son las siguientes (Welthungerhilfe, 2009):

- Etapa 1: Comprender el contexto del conflicto.

- Etapa 2: Análisis de los factores de división y fuentes de tensión.

- Etapa 3: Análisis de los factores de cohesión y de las capacidades locales para la paz.

- Etapa 4: Análisis del proyecto.

- Etapa 5: Análisis de los efectos y del impacto del proyecto.

- Etapa 6: identificar opciones y/o alternativas para el proyecto.

- Etapa 7: Comprobar las opciones y rediseñar el proyecto.

Estas etapas se articulan según la figura siguiente y cada paso implica un mayor conocimiento del contexto y una posibilidad de actuar teniendo en cuenta dicho contexto. 
Figura 6. Marco analítico de la acción sin daño

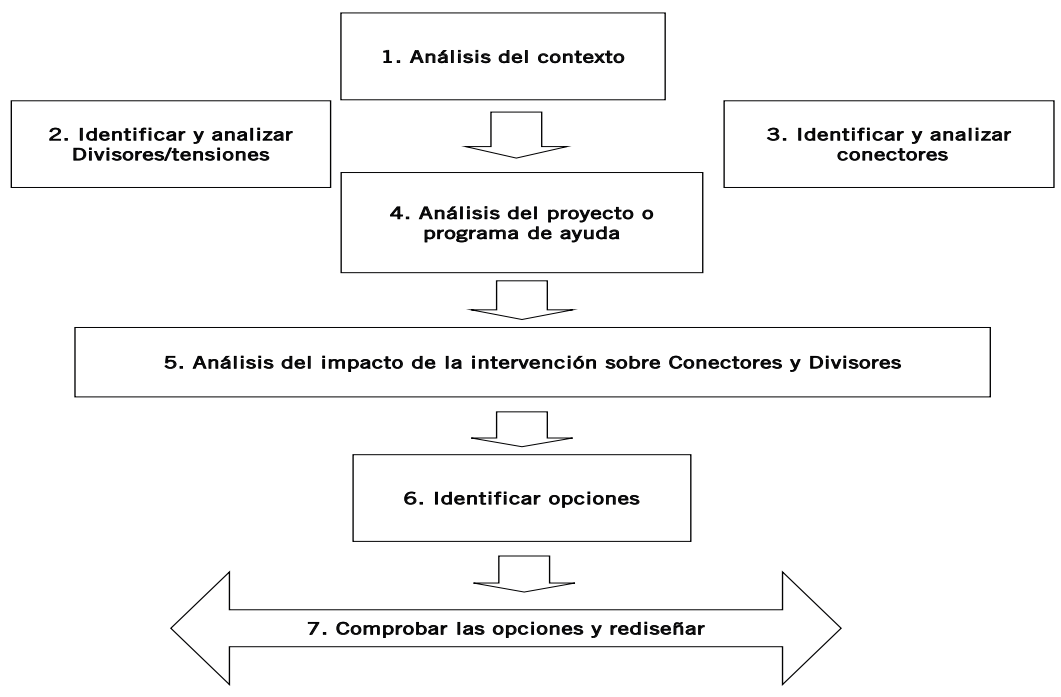

Fuente: Anderson (1999 y 2009), (Wallace, 2016) y elaboración propia.

El conocimiento del escenario donde se lleva a cabo el proyecto es fundamental para la Acción sin Daño y por ello constituye la primera etapa de la metodología. Las organizaciones que gestionan los proyectos y se erigen en titulares de responsabilidades han de conocer el terreno que pisan, los actores relevantes y las relaciones entre ellos.

Posteriormente, en las etapas 2 y 3 se analizan los denominados divisores y conectores.

Las situaciones de conflicto se caracterizan por tensiones y divisiones entre grupos. Estas situaciones también se caracterizan por la capacidad local para la paz y por los conectores que vinculan a la gente que participa en la lucha. Las cinco categorías que se usan en esta herramienta para analizar los conectores 0 divisores son los siguientes:

- Sistemas e instituciones.

- Las actitudes y las acciones.
- Valores e intereses.

- Experiencias comunes.

- Símbolos, ocasiones y eventos.

Estas categorías se analizan respecto a los conectores -lo que une a las personas y les lleva hacia el diálogo y la paz- y a los divisores y/o tensiones -lo que divide a las personas y les puede llevar al conflicto-. En la tabla 3 se muestran algunos ejemplos.

En la etapa 4, el análisis se enfoca en el diseño del proyecto, por tanto se estudian los elementos de la matriz y sus detalles. Se analizará el porqué del proyecto, cómo de implementará, por quién y para quién, dónde y cuándo y qué actividades se llevarán a cabo, entre otros elementos (Wallace, 2016; Anderson, 1999).

Y ello, para en la fase 5, identificar los impactos negativos y positivos que el proyecto tendrá sobre divisores y conectores. De esta forma, los gestores de las iniciativas deben conocer si las actividades 
Tabla 3. Ejemplos de categorías de conectores y divisores

\begin{tabular}{|c|c|c|}
\hline & $\begin{array}{l}\text { Capacidades para la paz y } \\
\text { conectores }\end{array}$ & Divisores \\
\hline Sistemas e instituciones & $\begin{array}{l}\text { En muchas de las sociedades en que } \\
\text { estalla una guerra civil, los mercados, } \\
\text { infraestructura y algunas instituciones } \\
\text { (como el sistema de comunicaciones, } \\
\text { p. ej.) acostumbran a actuar como } \\
\text { conectores de gente. Hoy en día, } \\
\text { además, Internet o los medios de } \\
\text { comunicación permiten a veces seguir } \\
\text { conectado con gente "del otro lado". }\end{array}$ & $\begin{array}{l}\text { Incluyen ejércitos o bandas, la } \\
\text { producción y distribución de armas, y } \\
\text { los aparatos de propaganda de guerra. } \\
\text { Además, muchas sociedades cuentan } \\
\text { con instituciones o sistemas que han } \\
\text { separado tradicionalmente los grupos } \\
\text { de gente provocando tensión entre } \\
\text { ellos (p. ej. Apartheid). }\end{array}$ \\
\hline Actitu & $\begin{array}{l}\text { En medio de una guerra siempre hay } \\
\text { gente que insiste en demostrar } \\
\text { tolerancia y amor, y que se niega a } \\
\text { demonizar o estereotipar a los "otros", } \\
\text { reconociendo incluso los errores y las } \\
\text { "malas prácticas" de su propio bando. }\end{array}$ & $\begin{array}{l}\text { Violencia, amenazas, tortura, } \\
\text { brutalidad, desplazamientos de } \\
\text { personas o expulsiones son algunos } \\
\text { ejemplos de acciones que dividen a la } \\
\text { gente. }\end{array}$ \\
\hline $\begin{array}{l}\text { Valores e intereses comunes } \\
\text { / distintos }\end{array}$ & $\begin{array}{l}\text { El amor a los niños, por ejemplo o, } \\
\text { incluso, la religión actúan a veces } \\
\text { como conector, así como el interés por } \\
\text { mantener un determinado sistema de } \\
\text { electricidad que beneficie a "todos los } \\
\text { bandos". }\end{array}$ & $\begin{array}{l}\text { Existen los llamados intereses de } \\
\text { grupo que persiguen mejorar su poder } \\
\text { o acceso a recursos de forma } \\
\text { particular. Asimismo, hay valores como } \\
\text { la religión, la etnia o la lengua que } \\
\text { pueden ser utilizados para separar. }\end{array}$ \\
\hline Experiencias comunes & $\begin{array}{l}\text { Una experiencia común, incluso si es } \\
\text { una experiencia de guerra, puede } \\
\text { servir como punto de partida para } \\
\text { conectar a gente enfrentada. }\end{array}$ & $\begin{array}{l}\text { Las diferentes percepciones del } \\
\text { mundo, así como sobre el bien/mal, lo } \\
\text { justo/injusto, proceden a menudo de } \\
\text { experiencias distintas. }\end{array}$ \\
\hline Símbolos y ocasiones & $\begin{array}{l}\text { Por ejemplo, el arte, la música, la } \\
\text { literatura, los aniversarios históricos o } \\
\text { las ceremonias pueden actuar a veces } \\
\text { de conectores. }\end{array}$ & $\begin{array}{l}\text { aAlgunos símbolos de subgrupos } \\
\text { odistintos pueden actuar también como } \\
\text { sdivisores, de forma que acentúen las } \\
\text { diferencias y provoquen suspicacias y } \\
\text { miedo entre los grupos. }\end{array}$ \\
\hline
\end{tabular}

Fuente: Anderson (1999 y 2009).

afectarán al incremento o a la disminución de la tensión o la violencia en el escenario de implementación del proyecto. Dicho impacto se producirá a través de la transferencia de recursos que todo proyecto implica y a través del comportamiento de los propios gestores del proyecto, que son igualmente titulares de responsabilidades. El comportamiento, además, trasmite una serie de mensajes éticos implícitos que pueden tener efectos negativos o positivos si implican una actitud de respeto, transparencia o rendición de cuentas o lo contrario (Wallace, 2016; Anderson, 1999).

Por último, en las etapas 6 y 7 se buscarán las mejores alternativas de acción y, si fuera necesario, se rediseñaría el proyecto de cara a disminuir las tensiones y reforzar las capacidades locales para la paz (Wallace, 2016; Anderson, 1999).

\section{La Acción sin Daño y el ciclo de proyecto bajo el enfoque de derechos}

Tanto la Acción sin Daño como el EML se pueden combinar para crear una metodología híbrida que permita a la Acción sin Daño participar de proyectos de desarroIlo, y al Enfoque de Marco Lógico tener 
Figura 7. Ciclo de vida del proyecto, marco lógico y Acción sin Daño

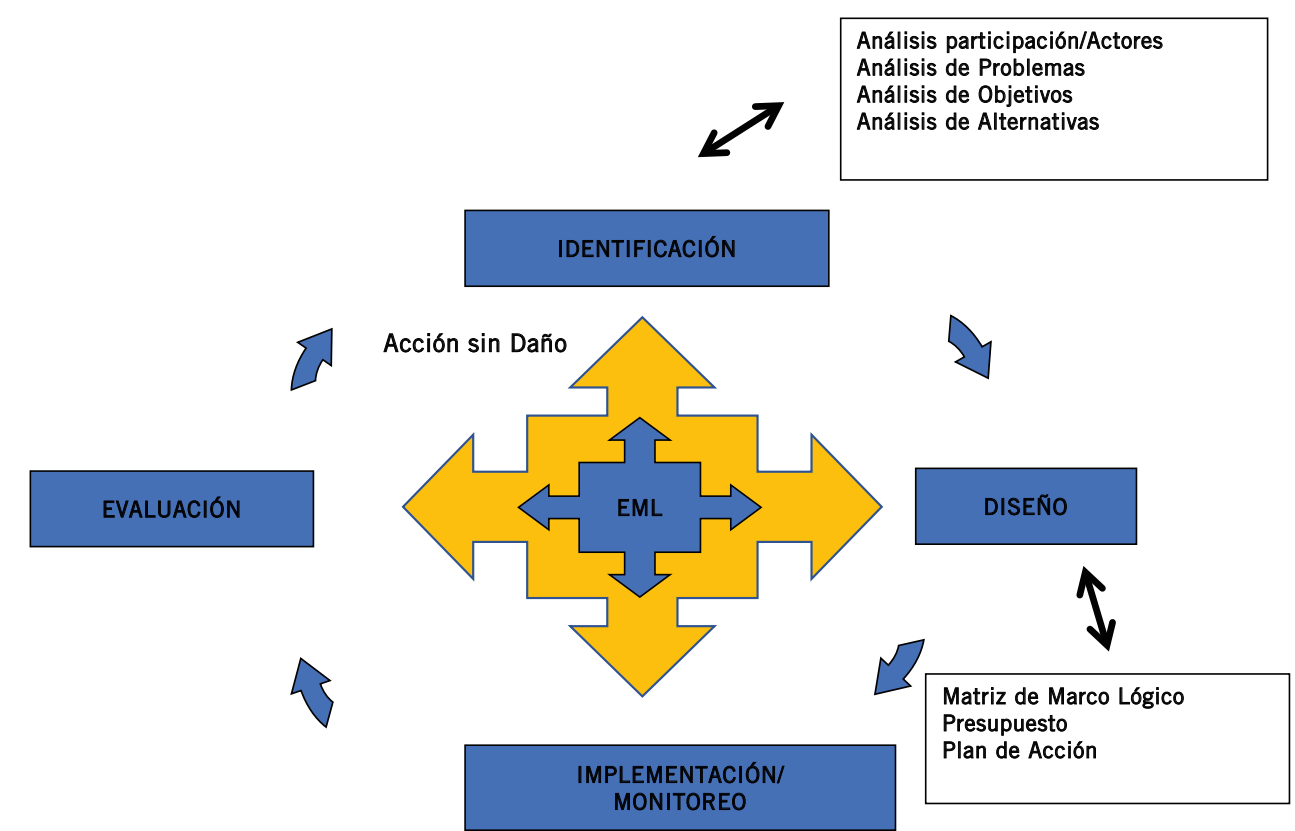

Fuente: Europe Aid Cooperation Office (2005) y elaboración propia.

en cuenta las situaciones de conflicto (ver figura 7). Ya que este artículo no pretende detallar la metodología a modo de manual, se presentarán unas pinceladas de cómo es posible congeniar ambas metodologías en las fases de identificación y diseño del ciclo de proyecto y respecto a las etapas 1-3 de la Acción sin Daño.

Como muestra la figura 8 , durante la fase de identificación del proyecto se plantea incluir en el análisis de participación del marco lógico las etapas 1-3 de la Acción sin Daño. De esta manera, se analiza el contexto teniendo en cuenta a los actores y cómo el comportamiento y la actitud de éstos en momentos determinados los puede convertir en divisores o conectores. Desde un primer momento se ha de tener en cuenta no sólo al actor en sí, sino a su papel según las circunstancias y cómo di- cho papel y su formar de actuar lo puede llevar a ser un divisor o conector. De esta manera, no se puede categorizar de manera genérica que un actor es de por sí conector o divisor, sino que en función de lo que hace en un momento determinado puede ser calificado como conector o divisor. En este sentido, algunos de los actores relevantes se tornarán en titulares de responsabilidades, deberes o derechos, por lo que es fundamental que ello se identifique para aproximar el EBDH. La población objetivo y beneficiaria se convierte en titular de derechos, mientras que la organización gestora del ciclo del proyecto lo será de responsabilidades, y en último caso es el Estado del lugar de implementación el titular de deberes u obligaciones.

Paralelamente, al iniciar el análisis de problemas y el árbol de problemas del marco 
Figura 8. Ciclo de vida del proyecto, marco lógico y Acción sin Daño combinados



Fuente: Europe Aid Cooperation Office (2005) y elaboración propia.

lógico se ha de tener en cuenta el comportamiento de los actores que los torna en divisores, ya que dicho comportamiento constituye una situación no deseable. Esto enriquecería el análisis básico de problemas, ya que dichos divisores se incluyen como problemas en el árbol de problemas. De la misma forma, a la hora de llevar a cabo el análisis de objetivos, la identificación de objetivos o soluciones se enriquecería con la incorporación de las capacidades locales para la paz o conectores, de manera que podrían ser incorporados como objetivos al árbol de objetivos. El papel de los actores como divisores y conectores tiene en cuenta el EBDH, por cuanto a que se identifican agentes titulares de derechos que tienen derecho a la asistencia a través del proyecto y no sólo que la merecen (ver tabla 1). Además, los actores que implementan el proyecto y sus socios tendrán la responsabilidad de prestar una asistencia correcta y "no provocar daño" con sus acciones, ya que son titulares de responsabilidades y, al menos, han de minimizar los impactos negativos de los proyectos.

Posteriormente, una vez llevado a cabo el análisis de alternativas y elaborada la matriz de planificación, durante la fase de diseño del proyecto, la Acción sin Daño puede ser nuevamente tenida en cuenta con la aplicación de las fases 4-7, tal y como ilustra la figura 8. En estas fases, se analizan los elementos de la matriz para ver los efectos que tendría el proyecto sobre una posible transferencia de recursos hacia los actores y los mensajes éticos implícitos que el proyecto podría trasmitir mediante el comportamiento de los titulares de la responsabilidad. De esta manera, la matriz de planificación puede ser probada de nuevo, teniendo en cuenta efectos de la misma sobre el contexto, 
que sólo a través del marco lógico básico pasarían desapercibidos. Por ejemplo, si un proyecto ofrece más recursos a un grupo social con respecto a otro, ello puede provocar efectos redistributivos negativos y violar los derechos de dicho grupo, por lo que el titular de la responsabilidad los gestores del proyecto - ha de ser consciente de ello. Por otro lado, también se pueden ofrecer mensajes éticos implícitos mediante la forma de vida o los valores diferentes de los gestores del proyecto, por lo que ello podría ser corregido para que no tenga efectos negativos sobre la gestión de las actividades, y de esta forma no influir negativamente sobre los beneficiarios del proyecto, que son titulares de derechos.

De esta manera, tanto en la fase de identificación como en la de diseño, la Acción sin Daño proporciona información fundamental que alimenta y mejora los análisis del Enfoque de Marco Lógico tradicional y ayuda a incorporar el EBDH.

\section{Conclusiones}

Como se ha visto en los apartados anteriores de manera breve, el EML y la Acción sin Daño son dos herramientas muy importantes para la gestión del ciclo de los proyectos. En el primer caso, para proyectos de desarrollo y en el segundo para la acción humanitaria. Sin embargo, la combinación de ambas metodologías aplicadas a proyectos de desarrollo es más que deseable y sería muy útil para aumentar la eficiencia de los proyectos en contexto de conflicto. Además, dicha combinación permite la aplicación del EBDH y concebir a los beneficiarios como titulares de derechos y a los gestores del ciclo de proyecto como titulares de responsabilidades.
La Acción sin Daño concluye con siete lecciones al aplicar su concepto y acudimos a ellas para presentar unas conclusiones donde se pone de manifiesto el EBDH (Wallace, 2016). Dichas lecciones son las siguientes:

1. Cada intervención o proyecto que entra en un conflicto pasa a formar parte de dicho contexto, por lo que afecta a los titulares de derec hos y son los titulares de la responsabilidad, como gestores del ciclo del proyecto, los que han de velar por la realización y la protección de los derechos humanos de sus titulares.

2. Todos los contextos se caracterizan por tener conectores y divisores, y son dichos elementos los que inciden sobre la realización o no de los derechos de los titulares de derechos.

3. Cada intervención está en interacción con los dos grupos de factores (divisores y conectores) de manera positiva, así como también negativa, a través de los elementos del proyecto.

4. Las intervenciones interactúan con los divisores y conectores a través de las acciones de la organización (transferencia de recursos) y del comportamiento del personal (mensajes éticos implícitos). Dichas acciones y comportamientos incidirán sobre la realización de los derechos de los titulares de derechos.

5. Los detalles de una intervención determinan el impacto sobre el contexto, por lo que la matriz de planificación definirá la manera en que los titulares de responsabilidad aseguran los derechos de los titulares de derechos o beneficiarios.

6. La experiencia muestra que siempre existen opciones alternativas que permitan la modificación de la matriz de 
planificación que implementarán los titulares de responsabilidad, y que afectará a la realización de los derechos de los titulares de derechos.

Estas lecciones no se tendrían en cuenta en un proyecto que no aplique la metodología sensible al conflicto como la Acción sin Daño, por lo que sería fundamental tenerla en cuenta, ya que un proyecto bien identificado y diseñado puede tener efectos negativos, si tan sólo se aplica una herramienta neutral como el marco lógico. Ser conscientes, al menos, de que existen factores de división y conexión en cualquier escenario social, no sólo puede mejorar la eficiencia de los proyectos, sino que además puede incrementar la seguridad de los beneficiarios y la realización de sus derechos como titulares de derechos. Además, la incorporación de la Acción sin Daño torna a los gestores del ciclo de proyecto en titulares de responsabilidad y permite aplicar de manera práctica algunos aspectos del EBDH.

\section{Bibliografía}

Anderson, Mary B. (1999). Do No Harm: How Aid Can Support Peace - Or War. Boulder: Lynne Rienner Publishers.

Anderson, Mary B. (2009). Acción sin daño: cómo la ayuda humanitaria puede apoyar la paz o la guerra. Tr. Jacques Mérat. Facultad de Ciencias Humanas, Programa de Iniciativas Universitarias para la Paz y la Convivencia- PIUPC. Bogotá: Universidad Nacional de Colombia.

EuropeAid Cooperation Office (2004): Aid Delivery Methods PCM. Project Approach Guidelines. Bruselas: European Commission.

Escobar, Arturo (2014). Sentipensar con la tierra: Nuevas lecturas sobre desarrollo, territorio y diferencia. Medellín: UNAULA.
Fernández Aller, Celia (coord.) et alia (2009). Marco teórico para la aplicación del enfoque basado en derechos humanos en la cooperación para el desarrollo. Madrid: Los Libros de la Catarata.

Gómez Galán, Manuel et alia (2013). El enfoque basado en derechos humanos en los programas y proyectos de cooperación. Madrid: CIDEAL.

NORAD (1999): The Logical Framework Approach (LFA). Handbook for objectiveoriented planning. $4^{\text {th }}$ etition, Oslo: Norwegian Agency for Development Cooperation (NORAD).

OHCHR (2006). Preguntas frecuentes sobre el enfoque de derechos humanos en la cooperación para el desarrollo. Ginebra: Oficina del Alto Comisionado de las Naciones Unidas para los Derechos Humanos (OHCHR).

Ortegón, Edgar et alia (2005). Metodología del marco lógico para la planificación, el seguimiento y la evaluación de proyectos y programas. Instituto Latinoamericano y del Caribe de Planificación Económica y Social (ILPES). Santiago de Chile: CEPAL. UNFPA (2010). A Human Rights-Based Approach to Programming. Practical Information and Training Materials. UNFPA and Harvard School of Public Health, Nueva York.

Wallace, Marshall (2016). From Principle to Practice. A User's Guide to Do No Harm. Cambridge: CDA Collaborative Learning Projects.

Welthungerhilfe (2009). Marco de orientación actuación sensible ante conflictos en la cooperación internacional. Bonn: Deutsche Welthungerhilfe.

Ziai, Aram. 2016. Development Discourse and Global History: From Colonialism to the Sustainable Development Goals. Londres: Routledge. 